\title{
User Equilibrium-Based Location Model of Rapid Charging Stations for Electric Vehicles with Batteries That Have Different States of Charge
}

\author{
Yong-Gwan Lee, Hyo-Seung Kim, Seung-Young Kho, and Chungwon Lee
}

\begin{abstract}
A model was developed for the location of rapid charging stations for electric vehicles (EVs) in urban areas, taking into account the batteries' state of charge and users' charging and traveling behaviors. EVs are one means of preparing for the energy crisis and of reducing greenhouse gas emissions. To help relieve range anxiety, an adequate number of $\mathrm{EV}$ charging stations must be constructed. Rapid charging stations are needed in urban areas because there is inadequate space for slow-charging equipment. The objective function of the model is to minimize EVs' travel fail distance and the total travel time of the entire network when the link flow is determined by a user equilibrium assignment. The remaining fuel range (RFR) at the origin node is assumed to follow a probabilistic distribution to reflect users' charging behavior or technical development. The results indicate that the model described in this paper can identify locations for charging stations by using a probabilistic distribution function for the RFR. The location model, which was developed on the basis of user equilibrium assignment, is likely to consider the congested traffic conditions of urban areas, to avoid locating charging stations where they could cause additional traffic congestion. The proposed model can assist decision makers in developing policies that encourage the use of EVs, and it will be useful in developing an appropriate budget for implementing the plan.
\end{abstract}

The electric vehicle (EV) is one of the most popular alternativefuel vehicles (1). However, range anxiety has restricted the pace at which EVs have penetrated the market. The construction of an adequate number of EV charging stations can help relieve range anxiety (2). In view of budget constraints, choice of the locations of such stations is important. Access to EV charging stations will affect the use rates of EVs, decisions concerning their use, the percentage of miles attained with electricity, the demand for petroleum, and power consumption at various times during the day $(3,4)$. Thus, the proper location of EV charging stations is essential, and some important studies have been conducted in the past few years (5-10).

To formulate a practical model for determining the appropriate locations for EV charging stations, several variables must be con-

Y.-G. Lee, S.-Y. Kho, and C. Lee, Department of Civil and Environmental Engineering, and H.-S. Kim, Integrated Research Institute of Construction and Environmental Engineering, Seoul National University, 1 Gwanak-Ro, Gwanak-Gu, Seoul, 151-744, South Korea. Corresponding author: C. Lee, chungwon@snu.ac.kr.

Transportation Research Record: Journal of the Transportation Research Board, No. 2454, Transportation Research Board of the National Academies, Washington, D.C., 2014, pp. 97-106.

DOI: $10.3141 / 2454-13$ sidered, including the vehicle range (VR), batteries' state of charge (SOC), users' charging behavior, and travel preferences. In the early stages of EVs, the targeted consumers were people who traveled almost exclusively within the urban area (11). In current models for locating EV charging stations in urban areas, slow-charging equipment was targeted, and the objectives of the studies were to optimize the total usage of electrical power, maximize profit, and minimize costs. The location of rapid charging stations in urban areas is also important because adequate space cannot be made available to accommodate the larger numbers of slow-charging locations that would be necessary. However, most studies are based on parking behavior, and research on en route charging is lacking. Rapid charging stations in urban areas can help increase accessibility to charging to a greater extent than such stations could in rural areas. Therefore, current planning involves establishment of charging stations first in urban areas and then expansion of their availability to intercity roads (12). However, rapid charging stations, at which EV users can recharge during their trips, have not been considered in most studies.

Flow-refueling location models (FRLMs) have been developed to find adequate locations for gasoline stations for vehicles that need to refuel during their trip. FRLMs for alternative-fuel vehicles are extended models of flow-capturing location models (FCLMs) that were developed for convenience stores $(13,14)$. An FCLM is a maximum covering model, and it cannot handle the multiple refueling stations needed for paths longer than the VR. The VR is the distance that a vehicle can travel when it is fully charged, and FRLMs can be extended by adding the VR constraint.

However, application of FRLMs to urban areas is difficult for two reasons. First, the travel paths used in these models were determined exogenously. In the existing models, all of the vehicles from the same origin-destination (O-D) pair must be assigned to one path. This is reasonable for intercity trips, but it would rarely occur in urban areas, because numerous alternative paths are available. In practice, drivers can detour to charge their vehicles if they so desire. Some studies have considered such detours, but only from the standpoint of the probability of trip availability $(15,16)$. Second, the SOC level was assumed to be 0.5 because only the marginal case of alternative-fuel vehicles was considered in the studies of FRLMs (17). If each vehicle has a different remaining fuel range (RFR), the path used by each vehicle may be different. RFR can be calculated by multiplying VR by the SOC level of the battery. When a constant RFR is used, the number of stations that should be constructed can be underestimated in urban areas.

To summarize the literature, two variations are required to develop the location model for EV charging stations in an urban area. One 
TABLE 1 Literature Review and Contribution of This Study $[5-10,14-24]$

\begin{tabular}{|c|c|c|c|c|c|}
\hline Study & Objective Function & Station & Spatial Scope & RFR & Travel Path \\
\hline Hodgson (14) & Minimum failure & $\mathrm{CS}$ & - & - & - \\
\hline Kuby and Lim (17) & Minimum failure & $\mathrm{AFV}$ & INT & $\mathrm{D}$ & EXO \\
\hline Kuby and Lim (18) & Minimum failure & AFV & INT & $\mathrm{D}$ & EXO \\
\hline Kuby et al. (19) & Minimum failure & AFV & INT & $\mathrm{D}$ & EXO \\
\hline Wang (10) & Minimum number of stations & $\mathrm{AFV}$ & - & $\mathrm{D}$ & EXO \\
\hline Upchurch et al. (20) & Minimum failure cost & $\mathrm{AFV}$ & INT & $\mathrm{D}$ & EXO \\
\hline Wang and Lin $(21)$ & Minimum number of stations & $\mathrm{AFV}$ & INT & $\mathrm{D}$ & EXO \\
\hline $\operatorname{Kim}(15)$ & Minimum failure & $\mathrm{AFV}$ & INT & $\mathrm{D}$ & EXO \\
\hline Ip et al. (9) & Minimum operational cost & EV & - & - & - \\
\hline Wang and Wang (22) & Minimum failure + construction cost & AFV & INT & $\mathrm{D}$ & EXO \\
\hline Hanabusa and Horiguchi (8) & Entropy maximization & $\mathrm{EV}$ & - & - & - \\
\hline Ge et al. (7) & Minimum users' loss & $\mathrm{EV}$ & - & - & - \\
\hline Wang et al. (23) & Maximum net income & BS & - & - & - \\
\hline Frade et al. (6) & Maximum covering & EV-S & INN & - & - \\
\hline Kim and Kuby (16) & Minimum failure & AFV & INT & $\mathrm{D}$ & EXO \\
\hline Capar and Kuby (24) & Minimum failure & $\mathrm{AFV}$ & INT & $\mathrm{D}$ & EXO \\
\hline Chen et al. (5) & Minimum access cost & EV-S & INN & - & - \\
\hline Proposed model & Minimum failure cost + network cost & EV-R & INN & $\mathrm{P}$ & ENDO \\
\hline
\end{tabular}

NOTE: $-=$ not considered; $\mathrm{CS}=$ convenience store; $\mathrm{AFV}=$ station for alternative-fuel vehicle $; \mathrm{EV}=$ station for electric vehicle; $\mathrm{BS}=$ battery switch station for EV; EV-S = slow-charging station for EV; EV-R = rapid charging station for EV; INT = intercity; INN = intra-city; $\mathrm{D}=$ deterministic $; \mathrm{P}=$ probabilistic; $\mathrm{EXO}=$ exogenously determined (all-or-nothing assignment); ENDO = endogenously determined (user equilibrium assignment).

is determination of the travel path endogenously, and the other is assumption of probabilistic RFR. In this way, the model can simulate EV users' behaviors reasonably and extend the results by enhancing batteries' volume or charging performances. The summary of the literature review and the contribution of this study are presented in Table 1.

The aim of the research reported in this paper is to develop a location model of rapid charging stations that considers vehicles' ranges, batteries' SOC, and users' charging and travel behaviors. A bilevel optimization model was developed in which the main problem was formulated as determining the locations of the stations and the patterns of use by EVs. The subproblem was formulated as determining link flow on the basis of the user equilibrium principle. To solve the problem in a reasonable time, a modified simulated annealing algorithm was proposed. The applicability of the model was tested in an example network.

\section{MODEL FORMULATION}

The proposed model is an uncapacitated facility location problem to minimize travelers' costs. The model is a modification of a $P$-median problem combined with a user equilibrium problem. The proposed model was based on the following considerations. First, the VR is assumed to be longer than the distance between the origin and the destination for all O-D pairs. This means that the EV's battery will not have to be recharged more than once during the trip. Thus, the VR constraint can be eliminated from the FRLM.

Second, the RFR at the origin node was assumed to follow a probabilistic distribution. The state of technical development or the supply of slow-recharging equipment at the origin node could affect the SOC. An EV that has a long range must be charged for a longer time than an EV that has a shorter range. If charging time becomes shorter or more slow-recharging installations are offered, the SOC level can be higher. If the RFR is assumed to be constant, as Capar and Kuby did (24), the RFR function is a unit impulse function. The cumulative distribution function of RFR, which is used in the literature, can be written as shown in Equation 1. In this study, the RFR function was assumed to be a probabilistic distribution function, such as a uniform distribution, an increasing distribution, or a triangular distribution. The distribution functions that were assumed in this study are shown in Equations 2 through 4.

Constant function: $G(r)= \begin{cases}0 & \text { if } 0 \leq r<0.5 r_{V} \\ 1 & \text { if } 0.5 r_{V} \leq r \leq r_{V}\end{cases}$

Uniform function: $G(r)=\frac{1}{r_{V}} \times r$

Increasing function: $G(r)=\frac{1}{r_{V}^{2}} \times r^{2}$

Triangular function:

$$
G(r)= \begin{cases}\frac{2}{r_{V}^{2}} \times r^{2} & \text { if } 0 \leq r<0.5 r_{V} \\ \frac{4}{r_{V}} \times r-\frac{2}{r_{V}^{2}} \times r^{2}-1 & \text { if } 0.5 r_{V} \leq r \leq r_{V}\end{cases}
$$

where

$$
\begin{aligned}
r & =\mathrm{RFR}, \\
r_{V} & =\mathrm{VR}, \text { and } \\
G(r) & =\text { cumulative distribution function of } r .
\end{aligned}
$$




\begin{tabular}{|c|c|c|c|c|c|}
\hline & $\begin{array}{ll}9 & 9 \\
15 & (j)\end{array}$ & $\begin{array}{l}\text { Flow from } i t \\
\text { Path used fo } \\
\text { Path used fo }\end{array}$ & $\begin{array}{l}: 100 \\
\text { rip without } r \\
\text { rip with rech }\end{array}$ & $\begin{array}{l}\text { narging: } i \rightarrow \\
\text { ging: } i \rightarrow j(\mathrm{~s}\end{array}$ & on) $\rightarrow j$ \\
\hline & Distribution & $\begin{array}{l}\text { Constant } \\
\text { distribution }\end{array}$ & $\begin{array}{c}\text { Uniform } \\
\text { distribution }\end{array}$ & $\begin{array}{l}\text { Triangular } \\
\text { distribution }\end{array}$ & $\begin{array}{l}\text { Increasing } \\
\text { distribution }\end{array}$ \\
\hline & Trip failure & 0 & 35 & 25 & 13 \\
\hline & Trip success & 100 & 65 & 75 & 87 \\
\hline$T_{v}=20$ & Without recharging & 0 & 25 & 12 & 43 \\
\hline & With recharging & 100 & 40 & 63 & 44 \\
\hline & Trip failure & 0 & 18 & 7 & 4 \\
\hline$r=40$ & Trip success & 100 & 82 & 93 & 96 \\
\hline$T_{v}=40$ & Without recharging & 100 & 62 & 71 & 85 \\
\hline & With recharging & 0 & 20 & 22 & 11 \\
\hline
\end{tabular}

FIGURE 1 RFR distribution functions and travel path by RFR.

The probabilities concerning how many EVs can travel with or without charging can be calculated by the RFR distribution function. Figure 1 shows the relationship between the type of RFR distribution function and the trip ratio. An increasing distribution may be found when people can easily charge their parked EVs. However, a uniform or a triangular distribution may be found when the slowcharging equipment installed is not sufficient to accommodate the requirements of the EVs.

Third, trips are classified by users' recharging behaviors. A fossilfuel vehicle can be refueled easily because many gasoline stations are available. There are far fewer charging stations for EVs than there are gasoline stations for fossil-fuel vehicles, so it was assumed that, before departure, the drivers of EVs chose where they would recharge their EVs. A user's behavior is determined on the basis of the RFR displayed in the vehicle's instrument panel. If the RFR is greater than the distance between the origin of the trip and its destination, the travelers can go to their destination without recharging, while they must locate a charging station or travel by using gasoline when the RFR is less than the distance to the destination. Here, two new decision variables, $\hat{y}_{i j k}$ and $\tilde{y}_{i j}$, are proposed to divide the trips into three groups. The decision variable $\hat{y}_{i j k}$ is the proportion of trips via station $k$ between origin $i$ and destination $j$. The decision variable $\tilde{y}_{i j}$ is the proportion that cannot travel by EV. Travelers who can no longer travel in their
EVs may travel by public transportation or by taxi, or they may incur the cost of emergency service to recharge their EVs. In the FRLM, travel paths are given as input data. As shown in Figure 2, three paths are available with the same distance from Node 1 to Node 8: 1-2-4-8, $1-5-4-8$, and 1-5-7-8. In the existing model, just one path is available among the three paths. Here, the consumption of the battery's charge is assumed to be proportional to the distance traveled because the RFR is displayed in kilometers, and users usually use this information to decide whether their EVs must be charged on their routes.

Finally, a traveler's trip follows the user equilibrium principle in terms of mean travel time, while the purpose of locating rapid charging stations is to minimize social costs, including travel time cost and the penalty associated with EV travel failure. The notation for formulating the model is as follows.

\section{Model Notation}

\section{Sets}

$N=$ node set, indexed by $n(N \ni n)$;

$\mathrm{A}=$ link set, indexed by $a(A$ э $a)$;

$I=$ set of origin nodes, indexed by $i(I \subseteq N, I \ni i)$;

\begin{tabular}{l}
\hline Travel path \\
$\begin{array}{c}\text { Path used when the } \\
\text { station is located at } \\
\text { Node 2 }\end{array}$
\end{tabular}

FIGURE 2 Travel paths by location of charging station. (RFR distribution function is assumed to be a uniform function. Link travel time is assumed to be constant.] 


$$
\begin{aligned}
J & =\text { set of destination nodes, indexed by } j(J \subseteq N, J \ni j) ; \\
H & =\text { set of paths connecting O-D pair } i, j, \text { indexed by } h(H \ni h) ; \text { and } \\
K & =\text { set of candidate nodes, indexed by } k \text { or } k^{\prime}\left(K \subseteq N, K \ni k, k^{\prime}\right) .
\end{aligned}
$$

\section{Number of Locations}

$P$ is the number of charging stations.

\section{Weights \\ $\gamma=$ additional penalty of failed travel and \\ $\omega=$ weight of $\gamma ; \omega=\gamma /(1+\gamma)$.}

Variables Concerning RFR

$$
r=\mathrm{RFR},
$$

$g_{i}(r)=$ probability distribution function of RFR on origin node $i$,

$G_{i}(r)=$ cumulative distribution function of $g_{i}(r)$, and

$\tilde{G}_{i j}=$ failure ratio for travel between O-D pair $i, j ; \tilde{G}_{i j}=G_{i}\left(c_{i j}\right)$.

\section{Node-Based Variables}

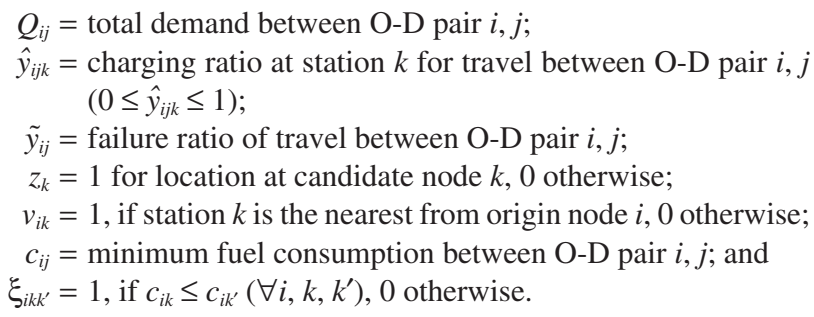

\section{Link-Based Variables}

$x_{a}=$ flow on link $a$,

$t_{a}=$ travel time on link $a$,

$c_{a}=$ fuel consumption on link $a$,

$\alpha_{a}, \beta_{a}=$ parameters of link $a$,

cap $_{a}=$ capacity of link $a$, and

$t_{a}^{0}=$ free-flow link travel time on link $a$.

\section{Variables Based on Node, Link, and Path}

$f_{h}^{i j}=$ flow on path $h$ between O-D pair $i, j$; and

$\delta_{a h}^{i j}=$ indicator variable; $\delta_{a h}^{i j}=1$, if link $a$ is on path $h$ between $\mathrm{O}-\mathrm{D}$ pair $i, j$, and 0 otherwise.

\section{Main Problem and Subproblem}

A bilevel optimization model is proposed. The main problem is one of location allocation to minimize the social cost, while the subproblem is one of trip assignment based on the user equilibrium principle. In the main problem, the EV trip failure ratio, the trip ratio via each station for each O-D pair, and the location of charging stations are determined. The equations were formulated on the basis of the general facility location problem (25). In the subproblem, link flow was determined. The equations in the subproblem are the modification of Beckmann's mathematical programming (26). The mathematical model is as follows.

Main problem:

$\min \left[\omega \sum_{i} \sum_{j} Q_{i j} c_{i j} \tilde{y}_{i j}+(1-\omega) \sum_{a} x_{a} t_{a}\left(x_{a}\right)\right]$

subject to

$\sum_{k} z_{k}=P$

$\hat{y}_{i j k} \leq z_{k} \quad \forall i, j, k$

$v_{i k} \geq \sum_{k^{\prime}} \xi_{i k k^{\prime}} z_{k^{\prime}}+z_{k}-P \quad \forall i, k$

$\tilde{y}_{i j} \geq v_{i k}\left[\tilde{G}_{i k} \xi_{i k j}+\tilde{G}_{i j}\left(1-\xi_{i k j}\right)\right] \quad \forall i, j, k$

$\tilde{y}_{i j}+\sum_{k} \hat{y}_{i j k}=\tilde{G}_{i j} \quad \forall i, j$

$\tilde{y}_{i j}+\sum_{k^{\prime}} \hat{y}_{i j h} \xi_{i k^{\prime} k}\left(1-\xi_{i k k^{\prime}}\right) \geq \tilde{G}_{i k} \xi_{i k j}+\tilde{G}_{i j} \xi_{i j k}\left(1-\xi_{i k j}\right) \quad \forall i, j, k$

$\hat{y}_{i j k} \geq 0 \quad \forall i, j, k$

$z_{k}, v_{i k}=0,1 \quad \forall i, k$

$x_{a}$ satisfies the subproblem $\quad \forall a$

Subproblem:

$\min \sum_{a} \int_{0}^{x_{a}} t_{a}(\chi) d \chi$

subject to

$\sum_{h} f_{h}^{i j}=Q_{i j}-\sum_{k} Q_{i j} \hat{y}_{i j k} \quad \forall i, j$

$\sum_{h} f_{h}^{i k}=\sum_{j} Q_{i j} \hat{y}_{i j k} \quad \forall i, k$

$\sum_{h} f_{h}^{k j}=\sum_{i} Q_{i j} \hat{y}_{i j k} \quad \forall k, j$

$x_{a}=\sum_{i} \sum_{j} \sum_{h} f_{h}^{i j} \cdot \delta_{a h}^{i j}+\sum_{i} \sum_{k} \sum_{h} f_{h}^{i k} \cdot \delta_{a h}^{i k}+\sum_{k} \sum_{j} \sum_{h} f_{h}^{k j} \cdot \delta_{a h}^{k j}$

$t_{a}(\chi)=t_{a}^{0} \cdot\left(1+\alpha_{a}\left(\frac{\chi}{\operatorname{cap}_{a}}\right)^{\beta_{a}}\right)$

The objective function (Equation 5) of the main problem is to minimize the weighted average of the network travel time and the EV trip failure penalty. Equation 6 stipulates that $P$ stations be located. Equation 7 states that any user traveling from origin $i$ to destination $j$ 
cannot be charged at station $k$ unless a charging station is located at node $k$. Equation 8 establishes constraints for finding the nearest station $k$ from origin node $i$. If station $k$ is the nearest station from origin $i$, the number of stations that are at the same distance or farther from node $i$ is $P$. Thus, if the value of the expression $\sum_{k^{\prime}} \xi_{i k k^{\prime}} z_{k^{\prime}}+z_{k}$ is $P+1$, station $k$ is the nearest station from origin $i$, and $v_{i k}$ becomes 1 . Equation 9 defines the travel failure ratio $\tilde{y}_{i j}$. If the nearest station from the origin is at the same distance as or closer than the destination, the travel failure ratio is the probability that RFR is shorter than the distance between the origin and the nearest station. If the nearest station from the origin is farther than the destination, the travel failure ratio is the probability that RFR is shorter than the distance between the origin and the destination. Equation 10 states that the sum of the travel failure ratio and the travel success ratio with charging at any of the stations is the same as $\tilde{G}_{i j}$, which is the proportion of EVs that cannot travel without charging from the origin to the destination. Equation 11 states that the probability of being unable to go from origin $i$ to station $k$ is equal to or smaller than the sum of the travel failure ratio and the travel success ratio with charging at any closer stations. Equation 12 states that the travel ratio has a nonnegative value. The constraints of Equation 13 are the integrality constraints. Link flows are determined in the subproblem.

The subproblem is the traffic assignment model with user equilibrium (Equations 15 through 20). To simulate the charging behavior, O-D flow is split into two groups; one group consists of those who travel from origin to destination directly (Equation 16), and the other group consists of those who travel and use charging stations (Equations 17 and 18). A trip with charging can be divided into two individual trips: one from origin to station (Equation 17) and another from station to destination (Equation 18). As a result, O-D flow with charging is double counted. The split ratio is determined at the level of the main problem. It is related to the user's charging behavior. If link travel time is not a function of link flow but instead is a constant, all the decision variables can be determined without the subproblem.

The objective function is formulated as a multiobjective function on the basis of the EV trip failure penalty and total travel time. Two objective functions are combined with weighting factor $\omega$. EV travelers tend to give up their trip with the EV if the detour cost is greater than the trip failure penalty. When $\omega$ approaches $0, \mathrm{EV}$ travelers decide their trip on the basis of travel cost. When $\omega$ approaches 1 , more travelers travel with their EV. If $\omega$ is 1 , the objective functions become the same as the performance indices of Upchurch et al. (20). Figure 3 shows the assignment results by varying $\omega$.

Since it is a combinatorial optimization problem, the location problem can be solved only through an enumeration technique. The computation complexity of an enumeration technique tends to increase exponentially as the feasible regions become larger. Many heuristic methods have been developed for solving location problems effectively, but they cannot be applied directly to the proposed model. The reason is that the gradient function or descent direction of the objective function cannot be calculated easily because the proposed model contains a subproblem with an objective function that is different from that of the main problem.

The objective function for each solution of location can be evaluated in polynomial time by the following steps. At the first time, the nearest station is calculated through use of the given $z_{k}$ and $P$ by Equation 21 .

$v_{i k}= \begin{cases}1 & \text { if } z_{k}-P+\sum_{k^{\prime}} \xi_{i k k^{\prime}} z_{k^{\prime}}=1 \\ 0 & \text { otherwise }\end{cases}$

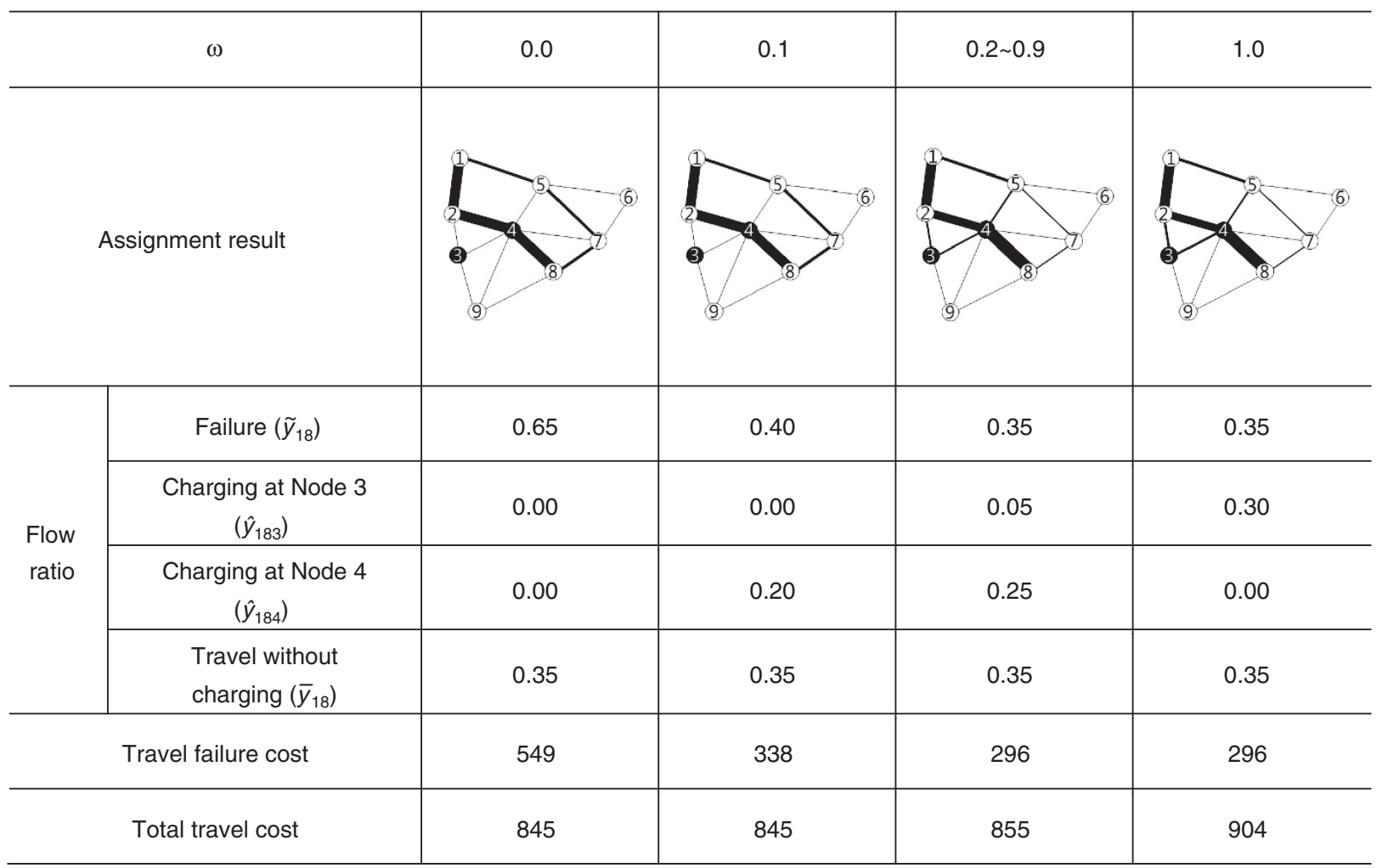

FIGURE 3 Assignment results by weight $\omega$. 
Then trip ratios $\left(\tilde{y}_{i j}, \hat{y}_{i j k}\right)$ can be determined by solving a simple linear programming problem, as shown in Equations 22 through 27. The link flows, which are used for evaluating the objective function, can be derived from the subproblem (Equations 15 through 20). The user equilibrium assignment problem is a convex nonlinear programming problem, and it can be solved by using the Frank-Wolfe algorithm (26).

$\min \left[\omega \sum_{i} \sum_{j} Q_{i j} c_{i j} \tilde{y}_{i j}+(1-\omega) \sum_{i} \sum_{j} \sum_{k} Q_{i j} \pi_{i j k} \hat{y}_{i j k}\right]$

subject to

$\hat{y}_{i j k} \leq z_{k}^{*} \quad \forall i, j, k$

$\tilde{y}_{i j} \geq v_{i k}^{*}\left[\tilde{G}_{i k} \xi_{i k j}+\tilde{G}_{i j}\left(1-\xi_{i k j}\right)\right] \quad \forall i, j, k$

$\tilde{y}_{i j}+\sum_{k} \hat{y}_{i j k}=\tilde{G}_{i j} \quad \forall i, j$

$\tilde{y}_{i j}+\sum_{k^{\prime}} \hat{y}_{i j h} \xi_{i k^{\prime} k}\left(1-\xi_{i k k^{\prime}}\right) \geq \tilde{G}_{i k} \xi_{i k j}+\tilde{G}_{i j} \xi_{i j k}\left(1-\xi_{i k j}\right) \quad \forall i, j, k$

$\hat{y}_{i j k} \geq 0 \quad \forall i, j, k$

where $z_{k}^{*}$ is given,

$\pi_{i j k}= \begin{cases}c_{i k}+c_{k j}-c_{i j} & \text { if } c_{i k}+c_{k j}>c_{i j} \\ 0 & \text { otherwise }\end{cases}$

and

$v_{i k}= \begin{cases}1 & \text { if } z_{k}-P+\sum_{k^{\prime}} \xi_{i k k^{\prime}} z_{k^{\prime}}=1 \\ 0 & \text { otherwise }\end{cases}$

To find a suitable solution, the objective function should be evaluated for every solution set. To do this efficiently, the modified simulated annealing algorithm was used to solve the proposed model. The solution algorithm is shown in Figure 4. To make the algorithm function more efficiently, the cooling and annealing schedules were controlled on the basis of repetitive works.

\section{APPLICATION AND RESULTS}

The validity and applicability of the proposed model were checked on the modified Sioux Falls, South Dakota, 24-node network, which was first used by LeBlanc et al. (27). This network is not considered to be realistic, but it has been used in many publications to debug code or examine the formats of data (28). The maximum distance between the origin and the destination was $12.1 \mathrm{~km}$ in the original network data, so the test network was enlarged to five times its original distance. Figure 5 summarizes the information on the base condition.

The main differences between the existing model and the proposed model were the RFR assumption and the way in which the recharging station was chosen. In the existing model, every vehicle had the same SOC and can be recharged only at the station on the shortest path. Only a few studies have considered the possibility of taking a detour for recharging. The proposed model chooses the detour path for travel via the recharging station endogenously.

First, the solutions of the existing model and the proposed model were compared. Because the former's objective function was to minimize trip failure and the total travel cost was included in the proposed model, the solutions can be different. However, it is difficult to determine whether one solution is significantly better than the other.

When the RFR was assumed to be half of the vehicle range, RFR was $50 \mathrm{~km}$ in this application, and the distances of most trips were shorter than the RFR (Figure 6). Thus, only one station should be constructed, and such an assumption cannot simulate the travel pattern of daily trips in an urban area. This is why the probabilistic RFR distribution should be used. The proposed model was compared with the existing model under the assumption that the RFR follows the uniform distribution function.

The problem was solved by using the proposed model and the existing model based on the probabilistic RFR distribution function, and their results were compared (Figure 7). Model I is the existing model, in which only the shortest paths were available. By adding constraints $\pi_{i j k} \cdot \hat{y}_{i j k}=0$ for all $i, j, k$ to the main problem, paths longer than the shortest paths must be used. If the reduced cost from origin node $i$ to destination node $j$ via station $k$ is greater than $0\left(\pi_{i j k}>0\right)$, station $k$ is not used $\left(\hat{y}_{i j k}=0\right)$. If station $k$ is chosen $\left(\hat{y}_{i j k}>0\right)$, then station $k$ is on the shortest path from $i$ to $j$. Model II has a detour available for recharging.

The number of trip failures and the cost of trip failures were decreased by increasing the number of charging stations in both models. However, no significant pattern of decreasing or increasing network costs was found. The comparison of the two models indicated that, for the model that had a detour available, trip failure and trip failure cost were lower than they were for the other model, but the total travel time costs were worse. This means that the proposed model can reflect travelers' behaviors with regard to making detours to recharge so they can finish their trips in EV mode.

Finally, the solution was analyzed by the objective function. When the objective function was to minimize the number of trip failures or trip failure costs, the total travel time of the network tended to be larger. If the facilities were located on the basis of the objective function, including only the trip failure term, they were likely to make the traffic condition worse.

\section{CONCLUSIONS AND FUTURE RESEARCH}

The development of a model for locating rapid charging stations in urban areas was reported in this paper. The assumption made for RFR distribution helped to reflect the technology development or demand variations. Use of an assignment model that was user-based allowed charging and traveling behaviors to be considered reasonably. The modified simulated annealing algorithm was proposed to solve the problem in polynomial time. According to the comparison with existing methods, the proposed model produced more stable solutions for various inputs, while the other methods produced different solutions when the number of stations or the vehicles' ranges were changed.

The research has three significant implications. First, the present models for locating charging stations are only for slow-charging 


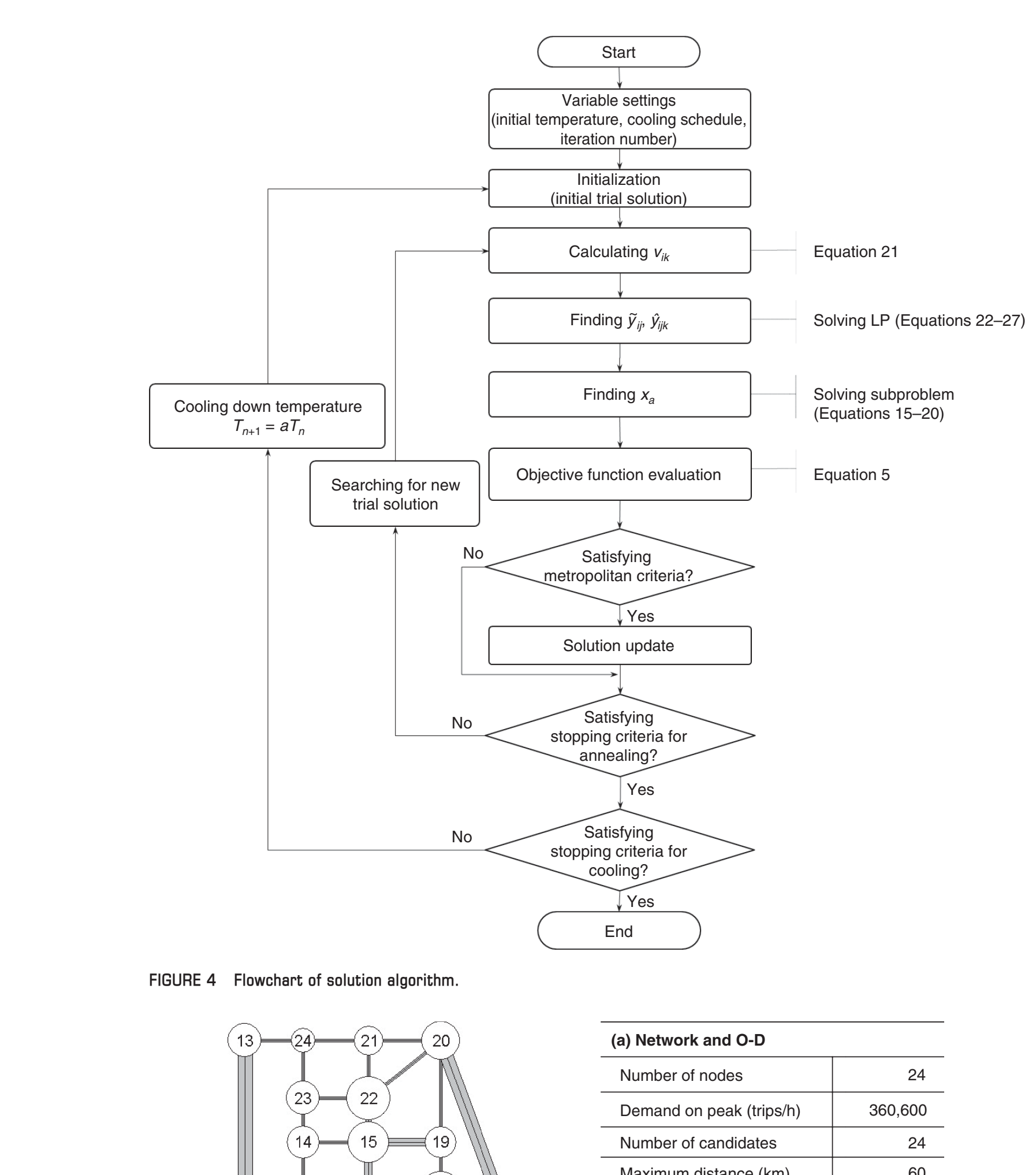

FIGURE 4 Flowchart of solution algorithm.

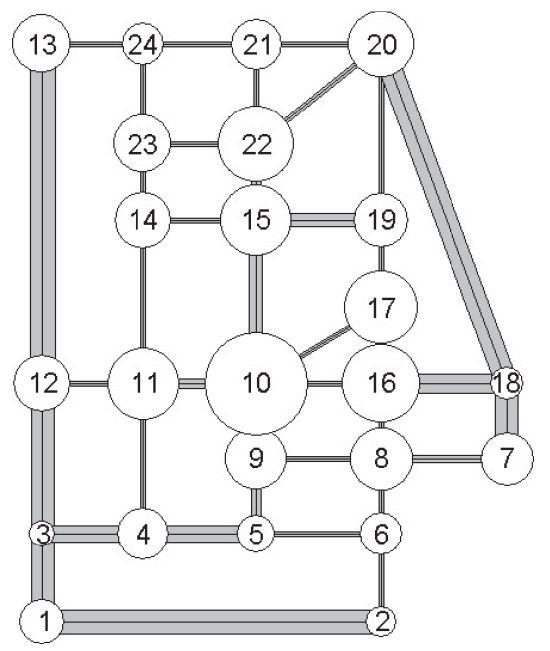

\begin{tabular}{l|r}
\hline \multicolumn{2}{l}{ (a) Network and O-D } \\
\hline Number of nodes & 24 \\
\hline Demand on peak (trips $/ \mathrm{h})$ & 360,600 \\
\hline Number of candidates & 24 \\
\hline Maximum distance $(\mathrm{km})$ & 60 \\
\hline
\end{tabular}

\begin{tabular}{|l|l|}
\hline (b) Input parameter & \\
\hline Weight $(\omega)$ & 0.5
\end{tabular}

\begin{tabular}{l|c}
\hline Weight $(\omega)$ & 0.5 \\
\hline (c) Travel time function & \\
\hline$\alpha_{a}=0.15, \beta_{a}=4.0$ & $\forall a$ \\
\hline
\end{tabular}

(d) RFR assumption

\begin{tabular}{l|r}
\hline Vehicle range $(\mathrm{km})$ & 60 \\
\hline Distribution function & Probabilistic \\
\hline
\end{tabular}

FIGURE 5 Parameters and input data. (Sizes of circles indicate departure volume from each node. Thicknesses of lines indicate capacity of each link.J 


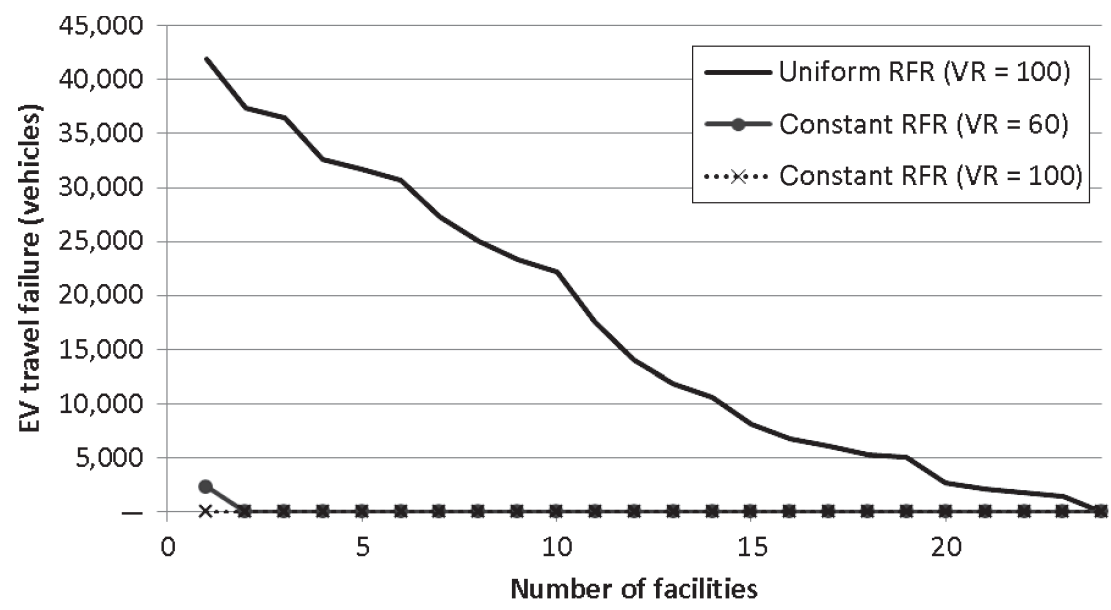

FIGURE 6 EV travel failure by assumption of RFR distribution function.

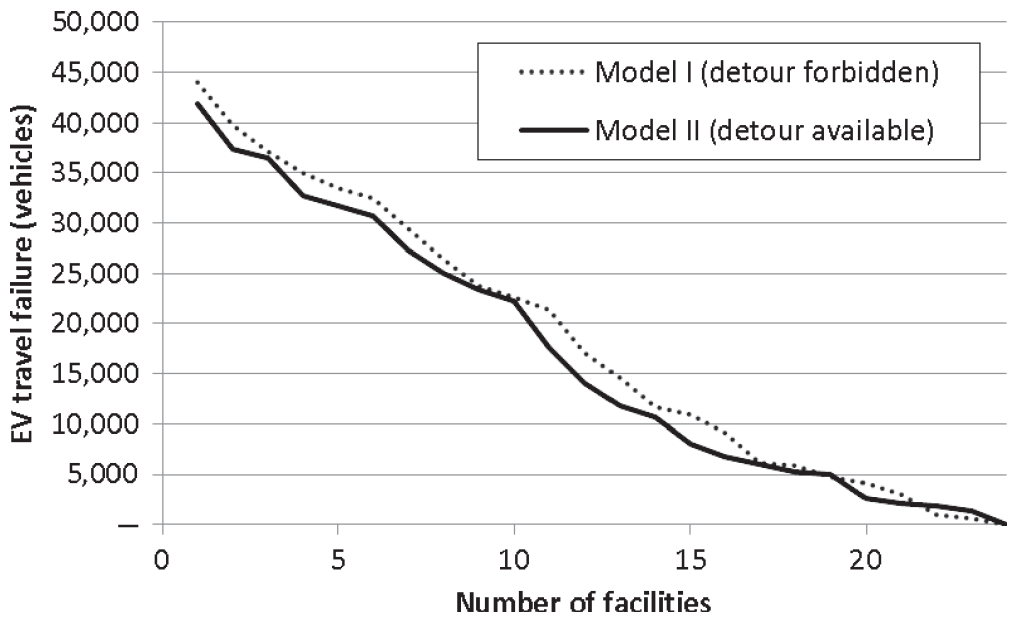

(a)

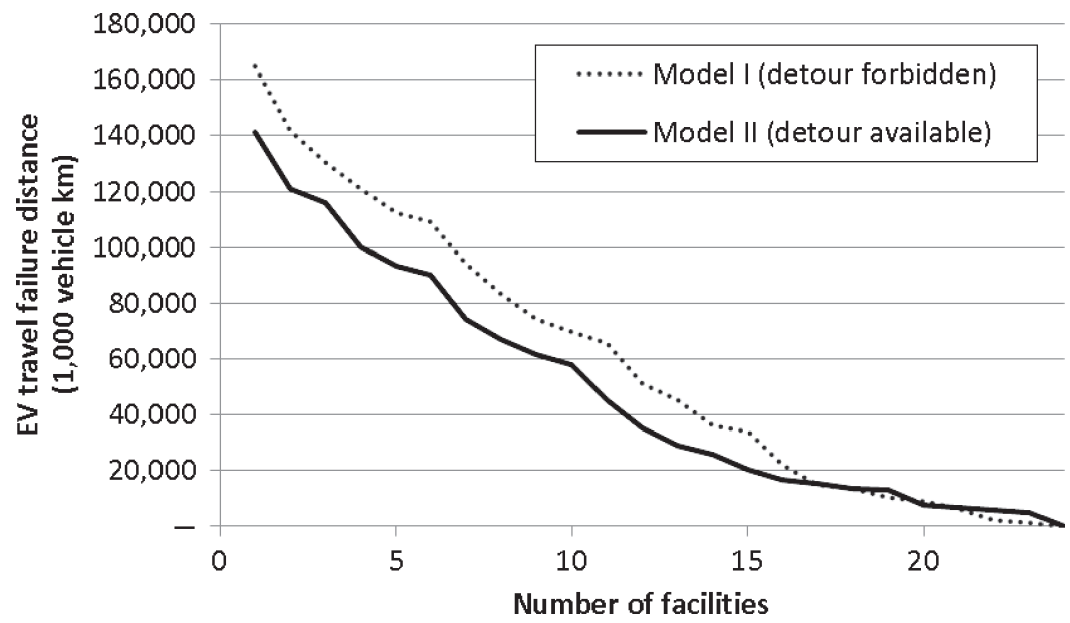

(b)

FIGURE 7 Comparisons of performance indices by detour possibility: (a) EV trip failure (vehicles) and (b) EV trip failure cost (vehicle kilometers).

(continued] 


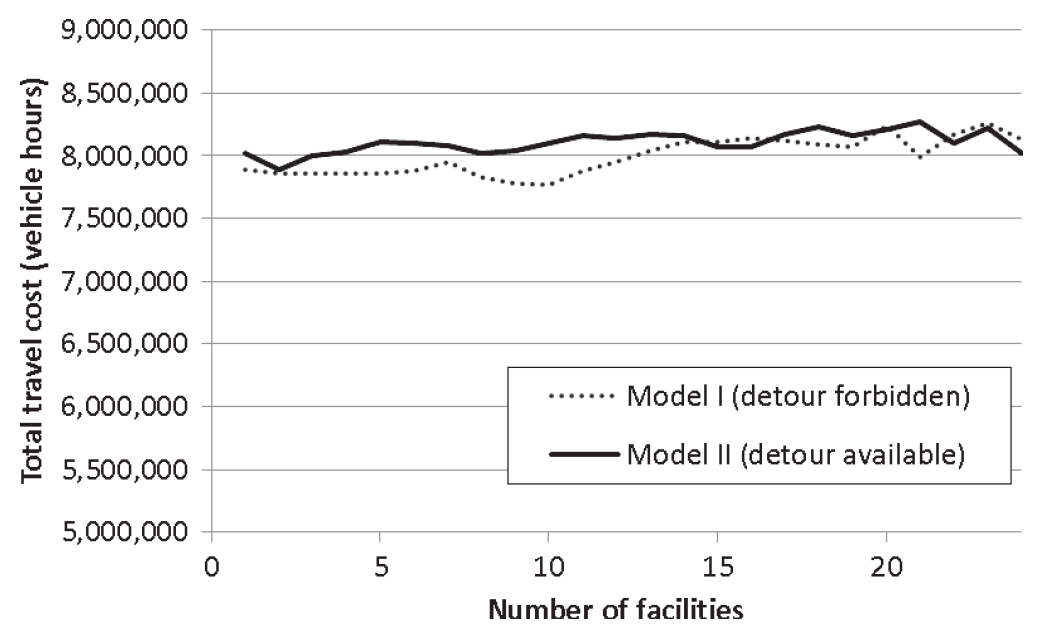

(c)

FIGURE 7 (continued) Comparisons of performance indices by detour possibility: (c) total travel cost (vehicle hours).

equipment, which can be installed in parking lots. To enhance the penetration of EVs, rapid charging stations are needed so that they can be used during trips. Therefore, a location model for rapid charging stations was developed. Second, the location model with constant RFR can produce different solutions when the vehicles' ranges are changed. This makes it difficult to determine definitively the best locations for EV charging stations when the technology is advancing rapidly. Therefore, the location model that was developed can contribute to determination of stable locations for charging stations by using a probabilistic RFR distribution function, even though the technology is advancing continuously. Third, the location model without the user equilibrium-based assignment problem is not likely to consider the congested traffic condition of urban areas. When only a few charging stations were installed during the EVs' introduction stage, many detours occurred for charging during trips, which can make traffic worse. This implies that the user equilibrium-based assignment should be included in the charging station location problem.

The model developed in this study provides a suitable solution for the location of rapid charging stations in an urban area because it reflects the probabilistic RFR distribution and the users' practical charging and traveling behaviors. To consider the viewpoint of decision makers, construction costs or operating costs can be included in the objective function. Therefore, the model provides a theoretical basis for determining suitable locations for rapid charging stations, and it can be applied directly to a real network by using the modified algorithm developed in this study.

The proposed model can be improved in further research and assessment work. If the RFR distribution function or battery consumption profile is surveyed in practice or predicted more reasonably, the proposed model can provide a more accurate solution for the targeted area without any modifications. The optimal number of stations can be evaluated when the model is extended to include the capacity of each station, charging speed, and the construction cost and operating cost of each station. By combining this model with the location of slow-charging stations or by considering vehicle mixing, various types of stations can be optimized, which is a subject for future research.

\section{ACKNOWLEDGMENTS}

The authors thank Dong-Kyu Kim of Seoul National University and the anonymous referees for their constructive and helpful comments. The generous support from the Integrated Research Institute of Construction and Environment at Seoul National University is also gratefully acknowledged.

\section{REFERENCES}

1. Boulanger, A. G., A. C. Chu, S. Maxx, and D. L. Waltz. Vehicle Electrification: Status and Issues. Proceedings of the IEEE, Vol. 99, No. 6, 2011, pp. 1116-1138.

2. Morrow, K., D. Karner, and J. Francfort. Plug-In Hybrid Electric Vehicle Charging Infrastructure. INL/EXT-08-15058. Idaho National Laboratory, Idaho Falls, 2008.

3. Chan, C. C. The State of the Art of Electric, Hybrid, and Fuel Cell Vehicles. Proceedings of the IEEE, Vol. 95, No. 4, 2007, pp. 704-718.

4. Lin, Z., and D. L. Greene. Promoting the Market for Plug-In Hybrid and Battery Electric Vehicles: Role of Recharge Availability. In Transportation Research Record: Journal of the Transportation Research Board, No. 2252, Transportation Research Board of the National Academies, Washington, D.C., 2011, pp. 49-56.

5. Chen, T.D., K.M. Kockelman, and M. Khan. Locating Electric Vehicle Charging Stations: Parking-Based Assignment Method for Seattle, Washington. In Transportation Research Record: Journal of the Transportation Research Board, No. 2385, Transportation Research Board of the National Academies, Washington, D.C., 2013, pp. 28-36.

6. Frade, I., A. Ribeiro, G. Gonçalves, and A. P. Antunes. Optimal Location of Charging Stations for Electric Vehicles in a Neighborhood in Lisbon, Portugal. In Transportation Research Record: Journal of the Transportation Research Board, No. 2252, Transportation Research Board of the National Academies, Washington, D.C., 2011, pp. 91-98.

7. Ge, S., L. Feng, and H. Liu. The Planning of Electric Vehicle Charging Station Based on Grid Partition Method. Presented at International Conference on Electrical and Control Engineering, Yichang, China, Sept. 16-18, 2011.

8. Hanabusa, H., and R. Horiguchi. A Study of the Analytical Method for the Location Planning of Charging Stations for Electric Vehicles. Presented at Knowledge-Based and Intelligent Information and Engineering Systems: 15th International Conference, Kaiserslautern, Germany, Sept. 12-14, 2011.

9. Ip, A., S. Fong, and E. Liu. Optimization for Allocating BEV Recharging Stations in Urban Areas by Using Hierarchical Clustering. Presented 
at 6th International Conference on Advanced Information Management and Service, Seoul, South Korea, Nov. 30-Dec. 2, 2010.

10. Wang, Y. Locating Battery Exchange Stations to Serve Tourism Transport: A Note. Transportation Research Part D, Vol. 13, 2008, pp. 193-197.

11. London's Electric Vehicle Infrastructure Strategy. Greater London Authority, Dec. 2009.

12. Wiederer, A., and R. Philip. Policy Options for Electric Vehicle Charging Infrastructure in C40 Cities. 2010. http://www.innovations.harvard edu/cache/documents/11089/1108934.pdf.

13. Berman, O., R. C. Larson, and N. Fouska. Optimal Location of Discretionary Service Facilities. Transportation Science, Vol. 26, No. 3, 1992 pp. 201-211.

14. Hodgson, M. J. A Flow-Capturing Location-Allocation Model. Geographical Analysis, Vol. 22, No. 3, 1990, pp. 270-279.

15. Kim, J. G. Location of Refueling Stations for Alternative Fuel Vehicles Considering Driver Deviation Behavior and Uneven Consumer Demand: Model, Heuristics, and GIS. PhD dissertation. Arizona State University, Tempe, 2010.

16. Kim, J., and M. Kuby. The Deviation-Flow Refueling Location Model for Optimizing a Network of Refueling Stations. International Journal of Hydrogen Energy, Vol. 37, 2012, pp. 5406-5420.

17. Kuby, M., and S. Lim. The Flow-Refueling Location Problem for Alternative-Fuel Vehicles. Socio-Economic Planning Sciences, Vol. 39 2005, pp. 125-145.

18. Kuby, M., and S. Lim. Location of Alternative-Fuel Stations Using the Flow-Refueling Location Model and Dispersion of Candidate Sites or Arcs. Networks and Spatial Economics, Vol. 7, 2007, pp. 129-152.

19. Kuby, M., L. Lines, R. Schultz, Z. Xie, J. G. Kim, and S. Lim. Optimization of Hydrogen Stations in Florida Using the Flow-Refueling Loca- tion Model. International Journal of Hydrogen Energy, Vol. 34, No. 15 2009, pp. 6045-6064.

20. Upchurch, C., M. Kuby, and S. Lim. A Model for Location of Capacitated Alternative-Fuel Stations. Geographical Analysis, Vol. 41, 2009 , pp. 85-106.

21. Wang, Y., and C. Lin. Locating Road-Vehicle Refueling Stations. Transportation Research Part E, Vol. 45, 2009, pp. 821-829.

22. Wang, Y., and C. Wang. Locating Passenger Vehicle Refueling Stations. Transportation Research Part E, Vol. 46, 2010, pp. 791-801.

23. Wang, C., J. Yang, N. Liu, and Y. Mao. Study on Siting and Sizing of Battery-Switch Station. 4th International Conference on Electric Utility Deregulation and Restructuring and Power Technologies, Weihai, China, 2011, pp. 657-662.

24. Capar, I., and M. Kuby. An Efficient Formulation of the Flow Refueling Location Model for Alternative-Fuel Stations. Institute of Industrial Engineers Transactions, Vol. 44, No. 8, 2012, pp. 622-636.

25. Daskin, M. S. Network and Discrete Location: Models, Algorithms, and Applications. Wiley, New York, 1995.

26. Sheffi, Y. Urban Transportation Networks: Equilibrium Analysis with Mathematical Programming Methods. Prentice Hall, Inc., Englewood Cliffs, N.J., 1985

27. LeBlanc, L. J., E. K. Morlok, and W.P. Pierskalla. An Efficient Approach to Solving the Road Network Equilibrium Traffic Assignment Problem. Transportation Research, Vol. 9, No. 5, 1975, pp. 309-318.

28. Bar-Gera, H. Transportation Network Test Problems. http://www.bgu. ac.il/ bargera/tntp/. Accessed Oct. 13, 2013.

The Alternative Transportation Fuels and Technologies Committee peer-reviewed this paper. 